\title{
Dimethyl Sulfoxide
}

National Cancer Institute

\section{Source}

National Cancer Institute. Dimethyl Sulfoxide. NCI Thesaurus. Code C437.

A highly polar org anic liquid that is used widely as a chemical solvent and a free radical scavenger. It shows a range of pharmacological activity including analgesia and antiinflammation. Because of its ability to penetrate biological membranes, it is used as a vehicle for topical application of pharmaceuticals. It is also used to protect cells and tissue during cryopreservation and has been used to treat extravasation damage caused by anthracycline-based chemotherapy. 